\title{
Markup Languages-How to Structure Chemistry-Related Documents
}

\section{by Peter Murray-Rust and Henry S. Rzepa}

Although the use of markup languages in publishing goes back to the 1960 s when IBM introduced GML (Generalized Markup Language), which subsequently evolved into the standard SGML, most authors are nowadays more familiar with the more recent implementation, referred to as HTML (HyperText Markup Language). The rapid rise in the use of HTML in conjunction with the growth of the World Wide Web was in large measure due to its ease of use for achieving presentational and visual effect. However, its limitations as a mechanism for expressing precisely defined data and meanings were not always adequately recognized. These limitations meant that in areas such as molecular sciences where precise meanings are essential, a variety of often proprietary solutions continued to be used to define and manipulate molecular "data" and information. The publishing processes were seen as quite separate and the process of translating data, information, and knowledge into a published entity remained an activity requiring much human perception. It is also worth noting that the reverse process of converting the published materials back into usable data remained equally human intensive and hence expensive.

The need to reconcile these two extremes was recognized at the first World Wide Web conference in 1994. A solution gelled shortly after the conference as a remarkable communal effort resulted in the specification of extensible markup language or XML. The ultimate vision of XML, as described by Berners-Lee, is the creation of a "Semantic Web."1 The rationale for this impressive effort included the following:

- Provision of a more universal infrastructure for publishing

- Recognition that the use of XML will require subject-specific vocabularies called "ontologies" Ontology is defined as a description — such as a formal specification of a program-of the concepts and relationships that can exist for a software agent or a community of agents.

- Provision of a mechanism for enhancing quality ("validation")

- Promotion of the creation of dynamic hyper-documents

- Recognition of the need to be able to reuse components of documents for other purposes

- Provision of a mechanism for creating smart archives, in which the re-usable components (information objects) can be readily identified
- Creation of an infrastructure for underpinning the emerging areas of e-business

The extension to chemistry included, therefore, the creation of a new generation of ontologically rich, primary publication and a clear division of the respective roles of humans and software agents (robots). Thus, humans should be able to:

- Publish all their data automatically

- Eliminate errors from publications

- Use the published literature as a database

- Understand information from other domains

Robots should be able to:

- Analyze publications (on whatever scale)

- Create secondary publications

- Purchase chemicals

- Synthesize chemicals from literature

To achieve this, we argue that a number of prerequisites must be in place:

- Automatic data capture, especially from instruments. We note that in 30 years we have moved from using instruments that captured data often only in analogue form (chart paper) to using standard computers to capture and process data to most recently an increasing tendency for placing these computers online and connecting them to centralized data stores.

- Common ontologies for a specific community (e.g., molecular science)

- Ontologically guided authoring.

\section{Issues Involved in "Capturing” Chemistry}

The following extract ${ }^{2}$ from a typical science journal illustrates both how precisely data and information must be represented, but also how much human perception is required to translate this information (e.g., to a reproducible experiment or a mechanistic interpretation):

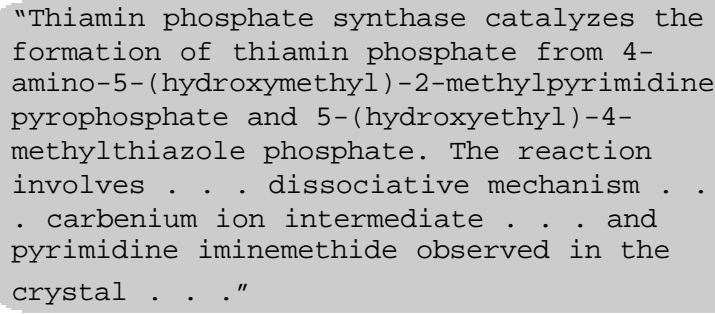


Note the profusion of chemical structure information, concepts, and terms, which only a trained human chemist could easily process. Quantitative concepts and units are also ubiquitous:

"A $500 \mu l$ aliquot of $0.8 \mu \mathrm{M}$ TP synthase in $50 \mathrm{mM}$ Tris-HCl (pH 7.5) and $6 \mathrm{mM} \mathrm{MgCl}_{2}$ incubated at room temperature with 50uM $\mathrm{CF}_{3} \mathrm{HMP}-\mathrm{PP}$."

An even greater degree of human perception is required when handling graphical chemical representations, which may contain many, often fuzzy and dangerous, human-only semantics (e.g., 2-D representations of 3-D properties, relative stereochemistry, aromaticity, hydrogen and other "weak" bonding, use of generic and "R" groups, reaction arrows, and mechanisms, etc.). The challenge, therefore, is to develop an infrastructure that can be routinely used to capture, store, and appropriately filter and display such information.

\section{The Current Position of XML}

As it is in 2002, XML offers a general, powerful, and extensible mechanism for handling both the "capture" and the publication of chemical information. In particular, XML allows for the first time this process to operate equally well in both directions. Our basis for stating this derives from the following observations:

- XML is increasingly accepted as an information infrastructure.

- The protocols are all public and many of the tools are open source.

- XML is vendor neutral, but with heavy vendor involvement.

- There is a large communal investment in generic tools (e.g., business2business, e-commerce).

- XML has a modular approach; an application is built from components.

- Domains are expected to create domain-specific XML protocols and tools.

- XML is increasingly universal in back-ends, middleware, and servers.

- Support for XML from database vendors is rapidly increasing.

- XML has close interoperability with other informatics standards such as UML, OMG/CORBA, etc.

- There is increasing support for "XML over the net" and from browsers (e.g., Internet Explorer, Netscape 6, etc).

- $\mathrm{XML}$ is very well supported by books, tutorials, etc.

\section{Global Open Activity in Scientific XML}

So how has the scientific community adopted these concepts? As noted above, the first World Wide Web conference specifically identified mathematics and chemistry as requiring specific markup languages. With this spark, CML (Chemical Markup Language) evolved between 1995-1997 to become the first scientific extended markup language. A concurrent effort lead to MathML becoming formalized as such in $1998 .^{3}$ We estimate that by 2002 , perhaps 50 specifically scientific applications have been described in some degree. For example, 37 scientific applications are quoted at $\langle w w w . x m l . c o m / p u b / r g / S c i e n c e>$ and a more general listing is at <www.oasis-open.org/ cover/xml.html\#applications $>$. The Science Citation Index shows around 570 references to the keyword XML, and SciFinder retrieves 38 references to the term "XML in chemistry."

\section{XML offers a general, powerful, and extensible mechanism for handling both the "capture" and the publication of chemical information.}

We also emphasize that XML is designed to allow markup languages to be combined, at whatever level of granularity, so that documents can contain any number of components deriving from specific XML languages. HTML, which we noted above, has evolved into one such language (XHTML), but in its latest development has been modularized into smaller, more easily implemented components (e.g., XFORMS, a data-entry and validation component can be implemented separately from other, more display-oriented components). XHTML can co-exist in a document with languages such as SVG (a scalable vector graphical language), MathML, and CML. We elaborate this when discussing namespaces (vide infra). ${ }^{4}$

\section{Some Essentials of an XML System}

The following tasks will have to be accomplished in order to implement an XML solution to publishing chemical information:

- Creation of documents from both legacy sources of data and de novo by humans

- Creation and capture of metadata (dictionaries of terms, tables of contents, codes, etc.)

- Specification of namespaces (a reserved addressing scheme for information)

- Human validation of the system (conformance to agreed specifications)

- Machine validation of documents (according to a specified and agreed upon schema)

- Document transformation (XSLT)

- $\quad$ Rendering and display (XSL-FO, domain-specific such as molecular representations) 
The design of an XML-based markup language should provide for the following:

- A simple, extensible document type definition (DTD) or schema (modular and not over-complicated)

- Agreed semantics

- One or more agreed and published ontologies

- Agreed examples and conformance tests

- A community of critical mass

Appropriate tools for accomplishing this should be identified. These might include the following:

- XML writers

- XML readers (more difficult than readers since the XML may not be normalized to a single form)

- Legacy converters (difficult because of variation and ambiguity in the original data which may require some degree of perception for an accurate conversion)

- Validators

- Dictionaries

- Editors

Custom-written XSLT style sheets and generic editors will accomplish some of these, but a document object model (DOM), which represents a syntax free abstraction of the data in memory, is probably essential for many subjects.

\section{Ontologies of Relevance to Chemistry*}

An overview of the types of ontologies required is shown in Table 1. Of the chemically specific information types, support should be included for:

- Molecules and substances

- Reactions

- Analytical information, especially spectra

- Computation and simulation (QM, mechanics, dynamics, etc.)

- "Data-centric" concepts (numbers, units, arrays, matrices, etc.)

- Specialist software for display, editing, searching, etc.

- "Adjoining" disciplines such as bio areas, materials science, etc.

\section{Creating Valid XML Documents}

Generic tools and protocols already exist to create valid XML documents. In particular, the use of DTDs (Document Type Definitions) and Schemas can bring enormous benefits, including eliminating/reducing software failure due to the use of invalid data and
Table 1: Types of Ontologies Relevant to XML in Chemistry and Tasks for the Chemical Community

General Non-Chemical Informatics

Business and commerce, gov- Reuse existing or emerging ernment, regulatory, academ approaches. ic, publishing, etc.

Domain-Specific Non-chemical

Mathematics (MathML), Collaborate to reuse existing healthcare (HL7/XML), or emerging approaches.

genomics (GeneOntology), etc.

Chemical-Specific but Generic Information Types

$\begin{array}{ll}\text { Numeric data, descriptive } & \begin{array}{l}\text { Create ontologies and reuse } \\ \text { prose, safety }\end{array} \\ \text { genic tools. }\end{array}$

\section{Chemical-Specific Information Types}

Chemical substances, Build the complete tool set. molecules, analytical and spectroscopic, reactions, computational chemistry

reducing difficulty of (human) understanding due to invalid publications. The DTD is a concept rooted in SGML, and is still used in XML to constrain the Markup vocabulary (i.e., the basic elements used for markup) and to some extent the (sub)structure of documents (i.e., what element can be a parent or child of another). Schemas are a more recent development, and unlike DTDs, are themselves expressed using XML. Of particular relevance to chemistry, they provide advantages over DTDs in that they can also be used for:

- Datatyping: numbers and user-defined types

- Enumeration (for example to specify the list of chemical elements)

- Lexical patterns

- Inheritance

Moreover, schemas allow for additional user-created rules (schematron/XSLT), and with dictionaries, support the conversion to software (e.g. CML-DOM), authoring (e.g., in editors), validation of the data on entry by the user.

\section{Namespaces-The Key to Making It Unique}

Each information object must be uniquely named to avoid collision and ambiguity. This is achieved using XML namespacing.

The example below shows a paragraph of text (derived from XHTML, which inherits the default namespace), within which components of CML are
*In this context, the term ontology refers to a machine readable set of definitions that create a taxonomy of classes and subclasses and relationships between them. Ref: <www.w3.org/2001/sw/WebOnt/charter> 
embedded, including prefixes using the defined namespaces:

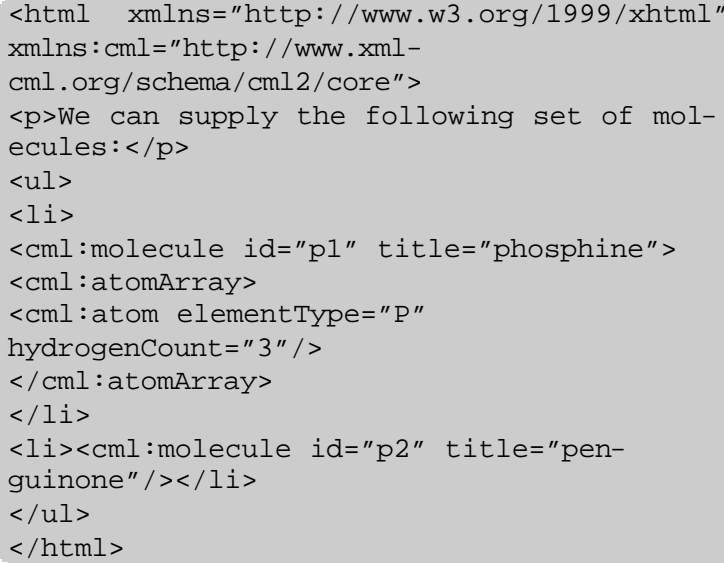

A proposal ${ }^{5}$ for domain-independent components for Scientific-Technical-Medical information, or STMML, contains key elements such as units, dictionary, metadata, item, array, and matrix and which supports datatypes such as numbers, $\mathrm{max} / \mathrm{min}$, ranges, errors, etc. The next example illustrates how CML can be used in conjunction with the STMML namespace ${ }^{5}$ to specify units and their constraints:

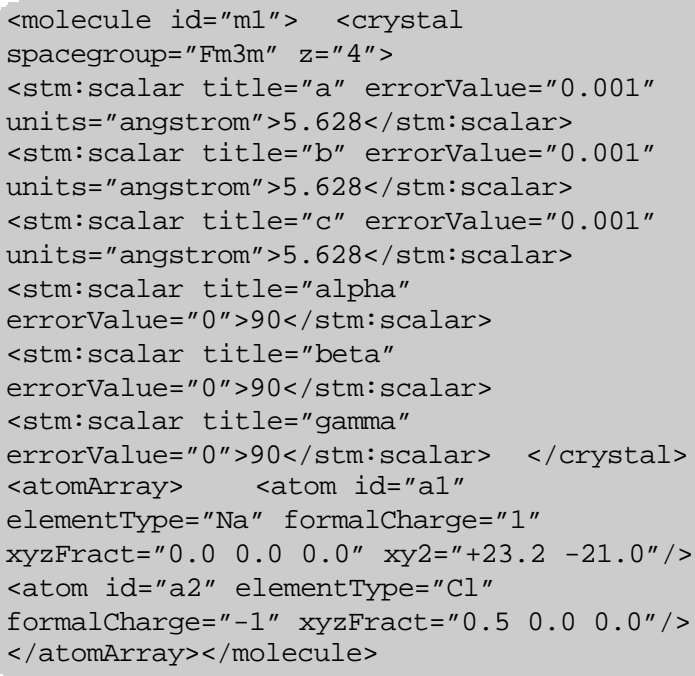

A more extended example of this concatenation of namespaces ${ }^{6}$ contains up to eight namespaced components and illustrates how a complete publication in XML/CML could be achieved. The use of namespaces can be seen in a more general context in Figure 1, which illustrates how the various specific XML components might relate to each other.

In particular, we note how the original CML specification $^{7}$ can be extended by modularization into a core namespace, and extended via other schemas into the following:

- CMLReact. A reaction, containing reactantLists, productLists and links between them.

- CMLComp. A container for computational and simulation input and results.

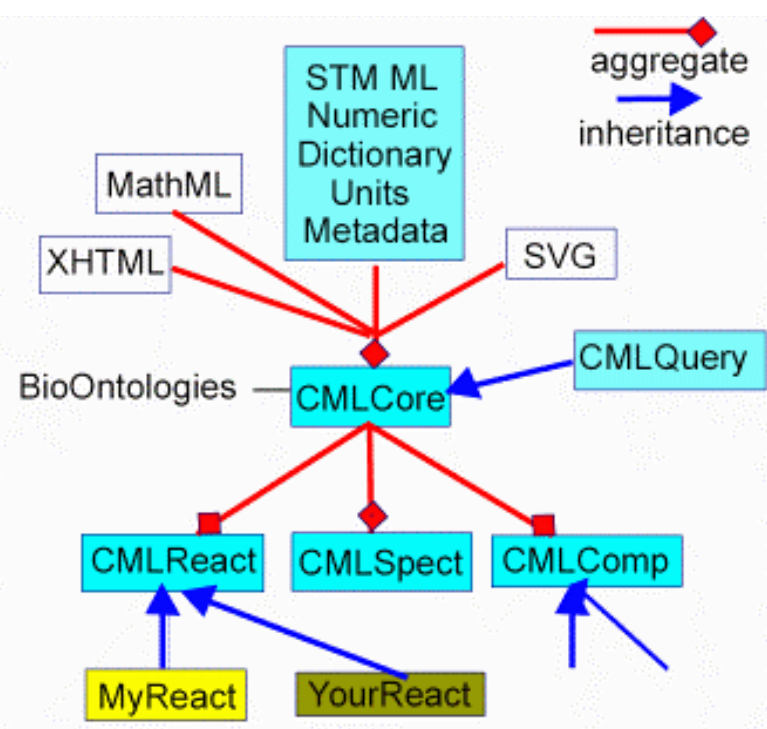

Figure 1: The use of namespaces in CML.

- CMLQuery. A generic query language.

- Hooks for other Schemas, such as SpectHook, for spectral parameters and data and links to molecular details (assignment).

\section{Dictionaries and Schemas}

It is useful to separate the domain ontology from the Schema/DTD, which allows the schema to be more abstract and which helps extensibility. Thus, with the instance document referring to NAMESPACE dictionaries, a three- or four-level hierarchy can be envisaged:

- The data instance

- The XMLSchema describing the instance

- The dictionary/ies describing the instance

- The schema describing the dictionaries

Such hierarchy and referring processes add semantics and ontology. An overview of this process is shown in Figure 2, where, for example, units are themselves verified by the UNITS dictionary.

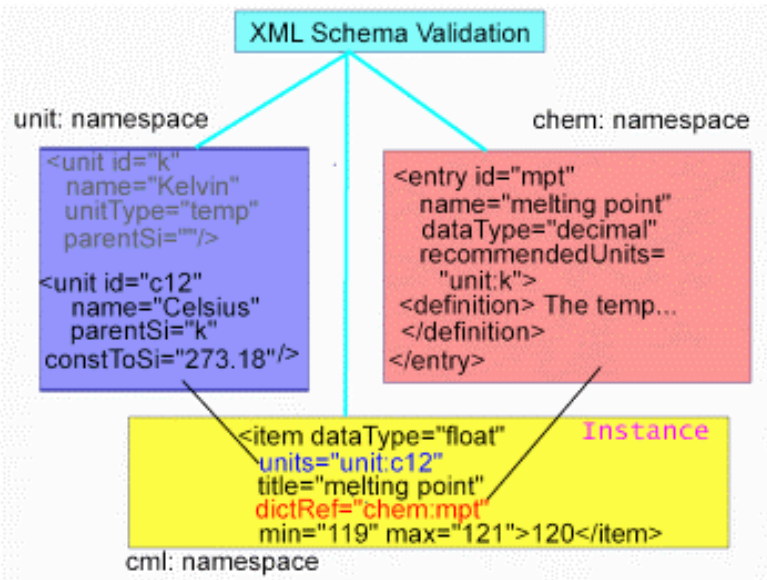

Figure 2: Validation scheme using dictionaries. 


\section{Document Structure and Metadata}

Common dictionaries and compendia usually have some of the following features:

- Dictionaries consist of curated entries and many are "flat" (e.g., the IUPAC GoldBook).

- Dictionaries are compiled within a single hierarchy:

- generic ("is A"):

eukaryote <-- vertebrate <-- mammal <-- human

- partitive ("has A"):

body <-- leg <-- foot <-- toe

- Dictionaries can now be associated with a namespace for uniqueness and navigation.

- Dictionaries must have curatorial information.

- Dictionaries should support versioning.

Metadata is an important component of a document or information object and it can serve a number of purposes:

- Navigational/Discovery - How is a piece of information to be discovered (e.g., Dublin Core and GILS)?

- Descriptive-What does the information mean and how is it to be used?

- Constraining-What constraints are there on the structure and content of the information? Is it valid? This would be accomplished using mainly XML Schemas.

- Supplementary-Additional (hyper-) data added from metadata

- Algorithmic-Deductions can be made from metadata (e.g., using Schematron, XSLT, and RDF).

- Chemical-descriptive-For example, medicinal, physical organic chemistry, Gold Book, stereochemistry.

- Chemical-constraining-For example, theoretical chemistry and CIF.

- Chemical-supplemental-For example, tables of atomic weights, dictionaries of compounds, etc.

- Chemical-algorithmic-For example, theoretical chemistry and CIF.

Communally agreed-upon schemas for defining such metadata are again seen as an essential component of the XML-infrastructures.

The existing IUPAC compendia provide a natural foundation for creating XML-based machine processible resources. They fall into three broad categories: descriptive (e.g., medicinal chemistry, physical organic chemistry, stereochemistry, etc.), validating (e.g., theoretical chemistry) and supplemental (e.g., atomic weights). Their availability for XML-based processes would be a considerable asset.

\section{Conclusions}

In this brief review of the application of XML in chem istry, we have summarized the essential advantages of adopting the XML approach. We have discussed in particular the benefits in creating reusable namespaced information components or objects, how these can be created and validated using subject-specific ontologies and dictionaries, and then how they can be enhanced with appropriate metadata. The role of communities and global organizations, such as IUPAC, is crucial to this endeavour. The use of such XML-based documents opens the prospect of creating avenues for the reversible flow of data and information between the scientific publication processes and the discovery, research, and learning processes in molecular sciences; a reversibility that has hitherto only been achieved with considerable human effort and expense.

\section{References}

1. T. Berners-Lee, M. Fischetti, M, Weaving the Web: The Original Design and the Ultimate Destiny of the World Wide Web, Orion Business Books, London, 1999.

2. D. H. Peapus, H. J. Chiu and N. Campobasso, Biochemistry, 2001, 40, 10103-10114.

3. See www.w3.org for details of all XML specifications.

4. G. V. Gkoutos, P. Murray-Rust, H. S. Rzepa, and M. Wright, Internet J. Chemistry, 2001, article 13.

5. P. Murray-Rust and H. S. Rzepa, 2002, submitted for publication. For the previous article in this series, see $\mathrm{P}$. Murray-Rust and H. S. Rzepa, Data Science 2002, 1, 8498.

6. P. Murray-Rust, H. S. Rzepa, and M. Wright, New J. Chem., 2001, 618-634.

7. P. Murray-Rust and H. S. Rzepa, J. Chem. Inf. Comp. Sci., 1999, 39, 928;

P. Murray-Rust and H. S. Rzepa, J. Chem. Inf. Comp. Sci., 2001, 1113;

G. Gkoutos, P. Murray-Rust, H. S. Rzepa, and M. Wright, J. Chem. Inf. Comp. Sci., 2001, 1124.

Peter Murray-Rust<pm286@cam.ac.uk> is a lecturer at the Unilever Centre for Molecular Informatics, Cambridge University, United Kingdom. Henry Rzepa <h.rzepa@ic.ac.uk> is a reader in the Department of Chemistry, Imperial College of Science, Technology, and Medicine, London, United Kingdom.

http://cml.sourceforge.net 